\title{
STOPPING AT THE MAXIMUM OF GEOMETRIC BROWNIAN MOTION WHEN SIGNALS ARE RECEIVED
}

\author{
X. GUO, ${ }^{*}$ Cornell University \\ J. LIU, ${ }^{* *}$ Yale University
}

\begin{abstract}
Consider a geometric Brownian motion $X_{t}(\omega)$ with drift. Suppose that there is an independent source that sends signals at random times $\tau_{1}<\tau_{2}<\cdots$. Upon receiving each signal, a decision has to be made as to whether to stop or to continue. Stopping at time $\tau$ will bring a reward $S_{\tau}$, where $S_{t}=\max \left(\max _{0 \leq u \leq t} X_{u}, s\right)$ for some constant $s \geq X_{0}$. The objective is to choose an optimal stopping time to maximize the discounted expected reward $\mathrm{E}\left[\mathrm{e}^{-r \tau_{i}} S_{\tau_{i}} \mid X_{0}=x, S_{0}=s\right]$, where $r$ is a discount factor. This problem can be viewed as a randomized version of the Bermudan look-back option pricing problem. In this paper, we derive explicit solutions to this optimal stopping problem, assuming that signal arrival is a Poisson process with parameter $\lambda$. Optimal stopping rules are differentiated by the frequency of the signal process. Specifically, there exists a threshold $\lambda^{*}$ such that if $\lambda>\lambda^{*}$, the optimal stopping problem is solved via the standard formulation of a 'free boundary' problem and the optimal stopping time $\tau^{*}$ is governed by a threshold $a^{*}$ such that $\tau^{*}=\inf \left\{\tau_{n}: X_{\tau_{n}} \leq a^{*} S_{\tau_{n}}\right\}$. If $\lambda \leq \lambda^{*}$ then it is optimal to stop immediately a signal is received, i.e. at $\tau^{*}=\tau_{1}$. Mathematically, it is intriguing that a smooth fit is critical in the former case while irrelevant in the latter.
\end{abstract}

Keywords: Optimal stopping problem; smooth fit; variational inequality

2000 Mathematics Subject Classification: Primary 60G40

Secondary 49L20

\section{Introduction}

In [15], Shepp and Shiryayev proposed and analyzed the problem of determining

$$
V_{\infty}(x, s)=\sup _{\tau} \mathrm{E}_{x, s}\left[\mathrm{e}^{-r \tau} S_{\tau}\right],
$$

where $r$ is a discount factor, $S_{t}=\max \left(\max _{0 \leq u \leq t} X_{u}, s\right)$ for some fixed $s \geq x=X_{0}, X_{t}$ is a geometric Brownian motion such that

$$
\mathrm{d} X_{t}=\mu X_{t} \mathrm{~d} t+\sigma X_{t} \mathrm{~d} W_{t},
$$

with $W_{t}$ a Wiener process, and $\tau$ is chosen among all choices of stopping time (the set of which is denoted by $\bar{B}$ ), meaning that no clairvoyance is allowed. They showed that the value function is finite if and only if $r>\mu$, and that the optimal stopping time $\tau^{*}$ is given by

$$
\tau^{*}=\inf \left\{t>0: X_{t} \leq a S_{t}\right\}
$$

Received 5 February 2002; revision received 13 February 2005.

* Postal address: School of Operations Research and Industrial Engineering, Cornell University, Ithaca, NY 14853,

USA. Email address: xinguo@orie.cornell.edu

** Postal address: School of Public Health, Yale University, New Haven, CT 06520, USA. 
where

$$
a=\left(\frac{\beta(\alpha-1)}{\alpha(\beta-1)}\right)^{1 /(\alpha-\beta)} .
$$

Here, $\alpha$ and $\beta, \alpha>1>0>\beta$, are the roots of

$$
-r+\mu x+\frac{1}{2} \sigma^{2} x(x-1)=0,
$$

given by

$$
\alpha=\frac{\frac{1}{2} \sigma^{2}-\mu+\sqrt{\left(\frac{1}{2} \sigma^{2}-\mu\right)^{2}+2 r \sigma^{2}}}{\sigma^{2}}, \quad \beta=\frac{\frac{1}{2} \sigma^{2}-\mu-\sqrt{\left(\frac{1}{2} \sigma^{2}-\mu\right)^{2}+2 r \sigma^{2}}}{\sigma^{2}} .
$$

Since payoffs of look-back options depend on the maximum or the minimum price of the derivatives achieved during the lifetime, this optimal stopping problem can be viewed as a perpetual look-back option pricing problem (see [4]).

In practice, however, stopping times may be discrete and choices of stopping time are limited. For instance, the sale of stocks or the exercising of options might be triggered by the (instant) arrival of information, such as news, insider information, rumor, misinformation, etc. This leads to the natural consideration of corresponding optimal stopping time problems over a smaller subset of $\bar{B}$. Dupuis and Wang [5] studied the problem of pricing perpetual American options with the choice of stopping time $B(\lambda)=\left\{\tau_{1}, \tau_{2}, \ldots\right\}$ such that $\tau_{1}, \tau_{2}-\tau_{1}, \ldots, \tau_{i}-\tau_{i-1}$ are independent and identically exponentially distributed with parameter $\lambda$. Rogers and Zane [14] studied liquidity issues in a similar setting. This particular subset of stopping times $B(\lambda)$ makes mathematical sense: if we assume that the arrival time of each piece of information follows an (appropriate) independent distribution, and that these distributions are identical, then it is well known that their superimposed process is Poisson.

Motivated by [5] and [14], we consider the problem of determining

$$
V^{*}(x, s, \lambda)=\sup _{\tau \in B(\lambda)} \mathrm{E}\left[\mathrm{e}^{-r \tau} S_{\tau}\right]
$$

where $S_{t}$ and $X_{t}$ are as given above and $B(\lambda)=\left\{\tau_{1}, \tau_{2}, \ldots\right\}$.

This is a discrete variant of (1), and may be viewed as a randomized version of the Bermudan look-back option pricing problem. Moreover, following the notion of randomized stopping [11], where the controller cannot stop the process but can increase the intensity of the stopping rate, this problem may be regarded as having a stopping rate between 0 and $\lambda$.

The solution structure reveals an intriguing aspect of this problem: there is a bifurcation in the form of the optimal policy depending on the rate $\lambda$. This is new and different from the results of [5] and [14] (as well as the earlier works of [1], [2], [3], [9], [12], [15], and [17]), where the conventional methodology of solving optimal stopping problems is to find the 'free boundary' between the 'continuation region' and the 'stopping region'. We, in contrast, show that there exists a threshold $\lambda^{*}$ such that if $\lambda>\lambda^{*}$, the optimal stopping problem is solved via the formulation of a free boundary problem for which the smooth-fit technique is applied; in this case the optimal stopping time $\tau^{*}$ is governed by a threshold $a^{*}$ such that

$$
\tau^{*}=\inf \left\{\tau_{n}: X_{\tau_{n}} \leq a^{*} S_{\tau_{n}}\right\}
$$

However, if $\lambda \leq \lambda^{*}$ then it is optimal to stop as soon as the first signal is received, i.e. at $\tau^{*}=\tau_{1}$. In the latter case, the derivation of the solution is independent of the smooth-fit principle; rather, 
it is derived by solving some 'variational inequalities'. As we will show, the Markov structure of $\left(X_{t}, S_{t}\right)$ and the linearity of the payoff function play a critical role throughout the analysis.

The solution structure has a simple intuitive interpretation. If signals arrive infrequently, one should stop as soon as possible because, in the presence of a discount factor, waiting for the next chance will take too long to get better rewards. On the other hand, if the signal is frequent then the constraint on stopping choices is less severe and provides sufficient flexibility in finding optimal stopping rules according to the classic method. The key reason for this type of bifurcation is that the utility function involves the running maximum $S_{t}$ instead of $X_{t}$; this is in contrast to the results of [5]. Note that $S_{t}$ no longer has an independent increment and increases only when $X_{t}=S_{t}$.

The structure of the paper is as follows: for both cases (frequent and infrequent signals), we present a proof using martingale theory, after a detailed derivation of the main theorem. At the end of the paper, we compare the asymptotic behavior of the solution when $\lambda \rightarrow \infty$ to that of the solution found in [15].

\section{Derivation of the main result}

We consider the problem of finding

$$
V^{*}(x, s, \lambda)=\sup _{\tau \in B(\lambda)} \mathrm{E}_{x, s}\left[\mathrm{e}^{-r \tau} S_{\tau}\right]
$$

where $X_{t}$ is given by (2) and the Poisson process with parameter $\lambda$ is independent of $X_{t}$.

As in [15], it is easy to see that the value function is infinite if $r \leq \mu$. Therefore, throughout the paper, we make the following assumption.

Assumption 1. We let $r>\mu$.

\subsection{Frequent signals}

2.1.1. Heuristic analysis. Note that the problem of determining (6) is a stopping time problem with state space $\{(x, s), x \leq s\}$. If we choose to stop at $\tau^{*}=\tau_{i}$ for some $i \geq 1$, then

$$
\begin{aligned}
\mathrm{d} X_{t} & =\mu X_{t} \mathrm{~d} t+\sigma X_{t} \mathrm{~d} W_{t}, & & t<\tau^{*}, \\
S_{t} & =S_{\tau_{i}}, & & t \geq \tau^{*} .
\end{aligned}
$$

(As such, one could view the process from a stochastic control perspective by introducing a binary control variable $u(t, \omega)$, with $u=1$ for $t<\tau_{i}$ and $u=0$ for $t \geq \tau_{i}$, as in [5]).

Intuitively, when signals are received frequently (we will determine later the critical 'threshold' of this frequency), we will have enough freedom to optimally choose between stopping and continuing. In this case, we can try to solve the problem using standard optimal stopping theory, by finding the free boundary $x=g(s)$ that separates the stopping region and the continuation region: when $x>g(s)$ we continue, and when $x \leq g(s)$ we stop immediately. Denoting this 'guessed' function by $V(x, s)$, we first analyze this function heuristically, and then prove its optimality.

In the continuation region (i.e. $\left.t<\tau^{*}\right), X_{t}>g\left(S_{t}\right)$ and

$$
\mathcal{L} V(x, s):=\lambda s-r V(x, s)+\mu x \frac{\partial V(x, s)}{\partial x}+\frac{1}{2} \sigma^{2} x^{2} \frac{\partial^{2} V(x, s)}{\partial x^{2}}=0,
$$

which is derived using the infinitesimal generator of geometric Brownian motion (see [10]). 
In the stopping region (i.e. $\left.t \geq \tau^{*}\right), X_{t} \leq g\left(S_{t}\right)$ and we have $\mathrm{d} S_{t}=0$ unless $X_{t}=S_{t}$, since any change in the nondecreasing process $S_{t}$ occurs only when $X_{t}=S_{t}$. Moreover, we can stop only when a signal is received, and the probability of receiving a signal in time $\Delta t$ is $\lambda \Delta t$, starting from time 0 . Thus, if we choose to stop at time $\Delta t$, we have

$$
\begin{aligned}
& V(x, s) \\
& \quad \geq \mathrm{e}^{-r \Delta t}\left(s \lambda \Delta t+(1-\lambda \Delta t) \mathrm{E}\left[V\left(X_{\Delta t}, S_{\Delta t}\right)\right]\right) \\
& \quad=\lambda s \Delta t+(1-r \Delta t)(1-\lambda \Delta t)\left(V(x, s)+\frac{\partial V(x, s)}{\partial x} \mu x \Delta t+\frac{1}{2} \sigma^{2} x^{2} \frac{\partial^{2} V(x, s)}{\partial x^{2}} \Delta t\right) .
\end{aligned}
$$

The equality holds only when the value function is optimal. Therefore, when $x \leq g(s)$, we see that

$$
\tilde{\mathcal{L}} V(x, s):=\lambda s-(\lambda+r) V(x, s)+\mu x \frac{\partial V(x, s)}{\partial x}+\frac{1}{2} \sigma^{2} x^{2} \frac{\partial^{2} V(x, s)}{\partial x^{2}}=0 .
$$

Equations (7) and (8) can be summarized in the form of a Hamilton-Jacobi-Bellman-type equation, as follows:

$$
\max (\tilde{\mathcal{L}} V(x, s), \mathcal{L} V(x, s))=0 .
$$

First, notice that the general form of the solution to $\mathcal{L} V(x, s)=0$ is

$$
V(x, s)=A(s) x^{\alpha}+B(s) x^{\beta},
$$

where $\alpha$ and $\beta$ are as in (4) and $A(s)$ and $B(s)$ are some functions to be determined. On the other hand, $\widetilde{\mathcal{L}} V(x, s)=0$ has a solution of the form

$$
V(x, s)=\frac{\lambda s}{\lambda+r}+C(s) x^{\bar{\alpha}}+D(s) x^{\bar{\beta}}
$$

where $\bar{\alpha}$ and $\bar{\beta}, \bar{\alpha}>1>0>\bar{\beta}$, are the roots of $-(r+\lambda)+\mu x+\frac{1}{2} \sigma^{2} x(x-1)=0, r>\mu$, given by

$$
\begin{aligned}
& \bar{\alpha}=\frac{\frac{1}{2} \sigma^{2}-\mu+\sqrt{\left(\frac{1}{2} \sigma^{2}-\mu\right)^{2}+2(r+\lambda) \sigma^{2}}}{\sigma^{2}}, \\
& \bar{\beta}=\frac{\frac{1}{2} \sigma^{2}-\mu-\sqrt{\left(\frac{1}{2} \sigma^{2}-\mu\right)^{2}+2(r+\lambda) \sigma^{2}}}{\sigma^{2}},
\end{aligned}
$$

and $C(s)$ and $D(s)$ are some functions to be determined. Note also that when $x=0$, the value function is finite, implying that $D(s)=0$.

Next, observe that

$$
\begin{aligned}
S_{\tau} & =\max \left(s, \sup _{0 \leq t \leq \tau} X_{t}\right) \\
& =\max \left(s, x \sup _{0 \leq t \leq \tau} \exp \left(\left(\mu-\frac{1}{2} \sigma^{2}\right) t+\sigma W_{t}\right)\right) \\
& =s \max \left(1, \frac{x}{s} \sup _{0 \leq t \leq \tau} \exp \left(\left(\mu-\frac{1}{2} \sigma^{2}\right) t+\sigma W_{t}\right)\right) .
\end{aligned}
$$

This suggests that the value function $V^{*}(x, s, \lambda)$ is linear, i.e. $V^{*}(x, s, \lambda)=s F(x / s)$ for some function $F$. Therefore, $V(x, s)$ should have the same property, and the boundary $x=g(s)$ should take the form $x=g(s)=a^{*} s$ for some threshold $a^{*}, 0 \leq a^{*} \leq 1$. 
In other words, we expect that

$$
\tau^{*}=\inf \left\{t>0: X_{t} \leq a^{*} S_{t}\right\}
$$

and

$$
V(x, s)= \begin{cases}C s\left(\frac{x}{s}\right)^{\bar{\alpha}}+\frac{\lambda}{\lambda+r} s, & x \leq a^{*} s \\ A s\left(\frac{x}{s}\right)^{\alpha}+B s\left(\frac{x}{s}\right)^{\beta}, & s \geq x \geq a^{*} s .\end{cases}
$$

Thus, to determine $V(x, s)$, we must find the unknown constants $A, B, C$, and $a^{*}$. To this end, we will apply the smooth-fit principle.

A smooth fit at $x=a^{*} s$, the boundary between the stopping region and the continuation region, according to (13) gives

$$
V(x+, s)=V(x-, s)=s
$$

and

$$
\frac{\partial V}{\partial x}(x+, s)=\frac{\partial V}{\partial x}(x-, s) .
$$

In addition, we have the following smooth condition at $x=s$ (see Remark 3, below):

$$
\left.\frac{\partial V(x, s)}{\partial s}\right|_{x=s}=0
$$

Combining (9)-(16) leads to

$$
\begin{aligned}
(1-\alpha) A+(1-\beta) B & =0, \\
C s\left(a^{*}\right)^{\bar{\alpha}}+\frac{\lambda s}{\lambda+r}=A s\left(a^{*}\right)^{\alpha}+B s\left(a^{*}\right)^{\beta} & =s, \\
\alpha A\left(a^{*}\right)^{\alpha}+\beta B\left(a^{*}\right)^{\beta} & =\bar{\alpha} C\left(a^{*}\right)^{\bar{\alpha}} .
\end{aligned}
$$

We find that

$$
a^{*}=\left(\frac{r \bar{\alpha} /(\lambda+r)-\beta}{r \bar{\alpha} /(\lambda+r)-\alpha} \frac{1-\alpha}{1-\beta}\right)^{1 /(\alpha-\beta)}
$$

and

$$
A=\frac{1}{\alpha-\beta}\left(\frac{r \bar{\alpha}}{\lambda+r}-\beta\right) a^{-\alpha}, \quad B=\frac{-1}{\alpha-\beta}\left(\frac{r \bar{\alpha}}{\lambda+r}-\alpha\right) a^{-\beta}, \quad C=\frac{r}{\lambda+r} a^{-\bar{\alpha}} .
$$

That is,

$$
V(x, s)= \begin{cases}\frac{s}{\alpha-\beta}\left(\left(\frac{r \bar{\alpha}}{\lambda+r}-\beta\right)\left(\frac{x}{a^{*} s}\right)^{\alpha}-\left(\frac{r \bar{\alpha}}{\lambda+r}-\alpha\right)\left(\frac{x}{a^{*} s}\right)^{\beta}\right), & x>a^{*} s, \\ \frac{\lambda}{\lambda+r} s+\frac{r}{\lambda+r} s\left(\frac{x}{a^{*} s}\right)^{\alpha}, & x \leq a^{*} s .\end{cases}
$$

Having solved $V(x, s)$ using the Hamilton-Jacobi-Bellman equation, we see that, in order for $V(x, s)$ to be a correct candidate for the value function, we should have $V(x, s) \geq s$ in the continuation region (i.e. $x>a^{*} s$ ), and $V(x, s) \leq s$ in the stopping region (i.e. $x \leq a^{*} s$ ). In fact, $V(x, s)$ satisfies the following variational relations. 
Proposition 1. The function $V(x, s)$ is $C^{2}$ on $(0, \infty) \times(0, \infty)$ except along the line $x=a^{*} s$, where it is $C^{1}$. Furthermore,

$$
\begin{array}{rlrl}
V(x, s) & \geq 0, & \\
V(x, s) & =s, & & x=a^{*} s, \\
\mathcal{L} V(x, s) & =0, & & x \geq a^{*} s, \\
\widetilde{\mathcal{L}} V(x, s) & =0, & & x \leq a^{*} s, \\
V(x, s) & \geq s, & & x \geq a^{*} s, \\
V(x, s) & \leq s, & & x \leq a^{*} s, \\
\frac{\partial V(x, s)}{\partial s} & =0, & & x=s .
\end{array}
$$

Proof. Of these relations, it remains to check that $V(x, s) \geq s$ for $x \geq a^{*} s$. Recalling that $V\left(a^{*} s, s\right)=s$, the conclusion is immediate, following from the facts that $V(x, s) \geq s$ for $x \geq a^{*} s$ and $V(\cdot, s)$ is an increasing function on $x \geq a^{*} s$.

We now show that our heuristic derivation of $V(x, s)$ is indeed the optimal value function $V^{*}(x, s)$ for large $\lambda$. This is accomplished by introducing the auxiliary value function $V_{0}(x, s)$, as in [5], where

$$
V_{0}(x, s)=\max (V(x, s), s) .
$$

It can be shown (see [5]) that $V_{0}(x, s)$ corresponds to the value function with the additional choice of stopping at 0 .

Theorem 1. When $r>\mu$ and $\lambda>2 r(r-\mu) / \sigma^{2}$, we have $V^{*}(x, s, \lambda)=V(x, s)$, where

$$
V(x, s)= \begin{cases}\frac{s}{\alpha-\beta}\left(\left(\frac{r \bar{\alpha}}{\lambda+r}-\beta\right)\left(\frac{x}{a^{*} s}\right)^{\alpha}-\left(\frac{r \bar{\alpha}}{\lambda+r}-\alpha\right)\left(\frac{x}{a^{*} s}\right)^{\beta}\right), & s \geq x \geq a^{*} s, \\ \frac{\lambda}{\lambda+r} s+\frac{r}{\lambda+r} s\left(\frac{x}{a^{*} s}\right)^{\bar{\alpha}}, & x \leq a^{*} s,\end{cases}
$$

with $a^{*}<1$ as in (17), $\alpha$ and $\beta$ as in (4), and $\bar{\alpha}$ and $\bar{\beta}$ as in (11). The optimal stopping time $\tau^{*}$ is given by

$$
\tau^{*}=\inf \left\{\tau_{n}: X_{\tau_{n}} \leq a^{*} S_{\tau_{n}}\right\}
$$

In particular, since $\tau_{n}-\tau_{n-1}$ has an exponential distribution with parameter $\lambda$ and is independent of $\tau_{i}, i \leq n-1,\left\{\mathrm{e}^{-r \tau_{n}} V_{0}\left(X_{\tau_{n}}, S_{\tau_{n}}\right)\right\}$ is a nonnegative supermartingale.

Before proving Theorem 1, several remarks are in order.

Remark 1. The smooth-fit principle in general requires at least $C^{1}$ smoothness across the boundary, but this is not sufficient for the principle to apply. One example in which the principle is not sufficient can be found in [7]. Furthermore, this principle is not necessary in solving our optimal stopping problem for small $\lambda$, as we will see later in this paper.

Remark 2. Both this paper and [16] extensively exploit the linearity of the value function in determining the free boundary $x=g(s)$. This is consistent with results of [6], which showed that the threshold-type solution structure of [16] depends on the linearity of the payoff function and the Markov structure of $X_{t} / S_{t}$. On the other hand, [7] gave an example of an optimal stopping problem with a nonlinear payoff function solved with a nonlinear free boundary. 
Remark 3. The smooth-fit condition in (16) is similar to the one used in [8], where such a condition involving suprema first appeared. There it was attributed to 'the extreme nature' of the value function.

2.1.2. Key steps in proving optimality: Theorem 1 . The proof of Theorem 1 combines the key ideas presented in [5] and [16], in addition to two simple technical lemmas.

Lemma 1. When $\lambda>2 r(r-\mu) / \sigma^{2}$, we have $0<a^{*}<1$.

Proof. From the expressions in (17), (4), and (11), it is clear that $\bar{\alpha}, \alpha>1$ and $\bar{\beta}, \beta<0$, and it suffices to show that

$$
\frac{r}{r+\lambda} \bar{\alpha} \leq 1<\alpha
$$

This holds when $(r /(r+\lambda)) \bar{\alpha} \leq 1$, which is equivalent to

$$
\sqrt{r^{2}\left(\frac{1}{2} \sigma^{2}-\mu\right)^{2}+2 r^{2}(r+\lambda) \sigma^{2}} \leq(r+\lambda) \sigma^{2}-r\left(\frac{1}{2} \sigma^{2}-\mu\right) .
$$

This, in turn, can be reduced to $\lambda>2 r(r-\mu) / \sigma^{2}$.

Remark 4. Evidently, if $\lambda \leq 2 r(r-\mu) / \sigma^{2}$ then $r \bar{\alpha} /(r+\lambda) \geq 1$.

Lemma 2. For $V(x, s)$ as given in (18), we have

$$
\mathrm{E}\left[\int_{0}^{T}\left|\frac{\partial V}{\partial x}\left(X_{t}, S_{t}\right)\right|^{2} \sigma^{2} X_{t}^{2} \mathrm{~d} t\right]<\infty .
$$

Proof. Since $V_{x}(x, s)$ is bounded by a constant, it suffices to prove that $\mathrm{E}\left[\int_{0}^{T} X_{t}^{2} \mathrm{~d} t\right]<\infty$. Notice that $\mathrm{E}\left[X_{t}^{2}\right]=x \mathrm{e}^{\mu t}<\infty$ for any $t, 0 \leq t \leq T$. Therefore, the proof follows from Fubini's theorem.

With these two lemmas, Theorem 1 can be proved by first verifying that $\mathrm{e}^{-r \tau_{n}} V_{0}\left(X_{\tau_{n}}, S_{\tau_{n}}\right)$ is a nonnegative, uniformly integrable supermartingale. Then, with this supermartingale, the optimality of the value function, i.e. $V^{*}(x, s)=V(x, s)$, is straightforward to prove using the optional sampling theorem [13] and the strong Markov property of $\left(X_{t}, S_{t}\right)$ : we simply modify the proof in Section 3 of [5] by replacing $V_{0}\left(S_{t}\right)$ and $\left(S_{t}-K\right)^{+}$(in the notation of [5]) with $V_{0}\left(X_{t}, S_{t}\right)$ and $S_{t}$, respectively.

For completeness, we present the proof in Appendix A.

\subsection{Infrequent signals}

If signals arrive infrequently, then the decision to stop has to be made in a timely manner. Otherwise, waiting for the next signal will take so long that the discount factor $\mathrm{e}^{-r t}$ will offset any gain in $S_{t}$. Intuitively, it is optimal to stop immediately the first signal arrives. Our analysis formalizes this intuition.

Consider the stopping strategy of $\tau=\tau_{1}$, for which the expected return is $f(x, s)=$ $\mathrm{E}_{x, s}\left[\mathrm{e}^{-r \tau_{1}} S_{\tau_{1}}\right]$. If it is suboptimal not to stop at $\tau_{1}$ for this value function, then an argument similar to those in the previous section yields

$$
-r f(x, s)+\mu x \frac{\partial f(x, s)}{\partial x}+\frac{1}{2} \sigma^{2} x^{2} \frac{\partial^{2} f(x, s)}{\partial x^{2}} \leq 0 .
$$


Moreover, since we do not have the freedom to stop immediately, we expect our return to be no larger than $s$ when we stop at $\tau_{1}$ (and to be even smaller if we continue). In short, we will look for a smooth function $f(x, s)$ that satisfies the following variational relations:

$$
\begin{aligned}
f(x, s) & \geq 0, \\
\mathcal{L} f(x, s) & \leq 0, \\
\widetilde{\mathcal{L}} f(x, s) & =0, \\
f(x, s) & \leq s, \\
\left.\frac{\partial f(x, s)}{\partial s}\right|_{x=s} & =0 .
\end{aligned}
$$

Proposition 2. The function

$$
Z(x, s)=\frac{\lambda}{\lambda+r} s\left(1+\frac{1}{\bar{\alpha}-1}\left(\frac{x}{s}\right)^{\bar{\alpha}}\right)
$$

is $C^{2}$ on $(0, \infty) \times(0, \infty)$ and solves the variational relation (19).

Proof. Solving $\widetilde{\mathcal{L}} Z(x, s)=0$ and using the linearity of the value function yields

$$
Z(x, s)=\frac{\lambda s}{\lambda+r}+C s\left(\frac{x}{s}\right)^{\bar{\alpha}},
$$

where $C$ is uniquely determined by $\left.(\partial Z(x, s) / \partial s)\right|_{x=s}=0$ to equal $\lambda /[(\lambda+r)(\bar{\alpha}-1)]$. With this solution, it is trivial to verify that indeed $\widetilde{\mathcal{L}} Z(x, s)=0$.

If we could show that $Z(x, s) \leq s$, then $\mathcal{L} Z(x, s) \leq 0$ would follow directly from $\tilde{\mathcal{L}} Z(x, s)=0$. To show that $Z(x, s)<s$, notice that $\bar{Z}(x, s)$ is an increasing function of $x$; therefore, it is sufficient to check that $Z(s, s) \leq s$. This translates into proving that $\lambda \bar{\alpha} /[(\lambda+r)(\bar{\alpha}-1)] \leq 1$, or, equivalently, that $r \bar{\alpha} /(\lambda+r) \leq 1$. This last inequality is immediate from Remark 4 with $\lambda \leq 2 r(r-\mu) / \sigma^{2}$.

Next, we show that $Z(x, s)$ is the optimal solution and is obtained by stopping at the first signal $\tau_{1}$.

Theorem 2. When $r>\mu$ and $\lambda \leq 2 r(r-\mu) / \sigma^{2}$, we have $V^{*}(x, s, \lambda)=Z(x, s)$, with $Z(x, s)$ as given in (20), and $\tau^{*}=\tau_{1}$.

Proof. The essence of the proof is similar to that when $\lambda>\lambda^{*}$. Define

$$
Y_{T}=\mathrm{e}^{-(r+\lambda) T} Z\left(X_{T}, S_{T}\right)+\int_{0}^{T} \lambda \mathrm{e}^{-(r+\lambda) t} S_{t} \mathrm{~d} t .
$$

Since $\mathrm{d} S_{t}=0$ (unless $X_{t}=S_{t}$ ) and $Z_{s}(s, s)=0$, by applying Itô's rule we see that $Y_{t}$ is a martingale.

Next, from (20), we clearly have

$$
\mathrm{E}\left[\int_{0}^{T}\left|\frac{\partial Z}{\partial x}\left(X_{t}, S_{t}\right)\right|^{2} \sigma^{2} X_{t}^{2} \mathrm{~d} t\right]<\infty .
$$


Thus, $Y_{T}$ is a martingale such that

$$
Z(x, s)=\mathrm{E}_{x, s}\left[Y_{0}\right]=\mathrm{E}_{x, s}\left[Y_{T}\right]=\mathrm{E}_{x, s}\left[\mathrm{e}^{-(r+\lambda) T} Z\left(X_{T}, S_{T}\right)\right]+\mathrm{E}_{x, s}\left[\int_{0}^{T} \lambda \mathrm{e}^{-(r+\lambda) t} S_{t} \mathrm{~d} t\right] .
$$

Note that $Z(x, s) \leq K s$ uniformly for a constant $K$ when $r>\mu$. Thus,

$$
\limsup _{T \rightarrow \infty} \mathrm{E}_{x, s}\left[\mathrm{e}^{-(r+\lambda) T} Z\left(X_{T}, S_{T}\right)\right] \leq K \mathrm{e}^{-(r+\lambda) T} \mathrm{E}\left[S_{T}\right] \leq K \mathrm{e}^{-(r+\lambda) T}\left(s+x \mathrm{e}^{\mu T}\right) \rightarrow 0
$$

when $r>\mu$. Hence, the monotone convergence theorem implies that

$$
Z(x, s)=\mathrm{E}\left[\int_{0}^{\infty} \lambda \mathrm{e}^{-(r+\lambda) t} S_{t} \mathrm{~d} t\right]
$$

and

$$
s \geq Z(x, s)=\mathrm{E}\left[\int_{0}^{\infty} \lambda \mathrm{e}^{-(r+\lambda) t} S_{t} \mathrm{~d} t\right]=\mathrm{E}_{x}\left[\mathrm{e}^{-r \tau_{n}} S_{\tau_{n}}\right] .
$$

Moreover, $\left\{\mathrm{e}^{-r \tau_{n}} S_{\tau_{n}}\right\}$ is a nonnegative, uniformly integrable supermartingale, following the analysis in the previous section.

Therefore, the optional sampling theorem [13] leads to

$$
s \geq \mathrm{E}_{x, s}\left[\mathrm{e}^{-r \tau_{1}} S_{\tau_{1}}\right] \geq \mathrm{E}_{x, s}\left[\mathrm{e}^{-r \tau_{N}} S_{\tau_{N}}\right],
$$

from which we obtain

$$
\mathrm{E}_{x, s}\left[\mathrm{e}^{-r \tau_{N}} S_{\tau_{N}}\right] \leq \mathrm{E}_{x, s}\left[\mathrm{e}^{-r \tau_{1}} S_{\tau_{1}}\right]=\mathrm{E}_{x, s}\left[\int_{0}^{\infty} \lambda \mathrm{e}^{-(r+\lambda) t} S_{t} \mathrm{~d} t\right]=Z(x, s),
$$

and $Z(x, s) \geq V^{*}(x, s, \lambda)$.

Finally,

$$
Z(x, s)=V^{*}(x, s, \lambda)
$$

is derived directly from (again) the strong Markov property of $\left(X_{t}, S_{t}\right)$ :

$$
\begin{aligned}
\mathrm{E}_{x, s}\left[\mathrm{e}^{-r \tau^{*}} S_{\tau^{*}}\right] & =\mathrm{E}_{x, s}\left[\int_{0}^{\infty} \lambda \mathrm{e}^{-r \tau^{*}} \mathrm{E}_{x, s}\left[\mathrm{e}^{-r \tau^{*}} S_{\tau_{*}} \mid \tau_{1}=t\right] \mathrm{d} t\right] \\
& =\mathrm{E}_{x, s}\left[\int_{0}^{\infty} \lambda \mathrm{e}^{-(r+\lambda) t} \mathrm{E}_{X_{t}, S_{t}}\left[\mathrm{e}^{-r \tau_{0}^{*}} S_{\tau_{0}^{*}}\right] \mathrm{d} t\right] \\
& =\mathrm{E}_{x, s}\left[\int_{0}^{\infty} \lambda \mathrm{e}^{-(r+\lambda) t} S_{t} \mathrm{~d} t\right] \\
& =Z(x, s),
\end{aligned}
$$

where $\tau_{0}^{*} \in B \cup\{0\}$.

\section{Comparison with Shepp and Shiryayev [15]}

We have solved the optimal stopping problem of determining (5) for a subset $B \not \subset \bar{B}$. It is interesting to compare our results with those of [15]. First, consider the following proposition.

Proposition 3. Direct calculations show that $a^{*} \geq a$, where a and $a^{*}$ are given by (3) and (17), respectively. 
This has a clear interpretation. The stopping rules in both cases (1) and (5) are based on the ratio $X_{t} / S_{t}$, except that the latter has a restriction on the choice of stopping times. With this constraint, we should be more 'conservative' and continue only when $X_{t}$ is closer to $S_{t}$ (i.e. $X_{t} / S_{t}$ is larger), so that the chance of getting an increase in $S_{t}$ is higher. Thus, $a^{*}>a$.

Moreover, if the freedom to stop is restricted, then the corresponding optimal value function is naturally smaller than that obtained without restrictions.

Proposition 4. We have $V^{*}(x, s, \lambda) \leq V_{\infty}(x, s)$, where $V_{\infty}(x, s)$, given in [15], is such that

$$
V_{\infty}(x, s)= \begin{cases}\frac{s}{\alpha-\beta}\left(-\beta\left(\frac{x}{a s}\right)^{\alpha}+\alpha\left(\frac{x}{a s}\right)^{\beta}\right), & s \geq x \geq a s, \\ s, & x \leq a s .\end{cases}
$$

Proof. For $\lambda \leq 2 r(r-\mu) / \sigma^{2}$, this proposition is obvious, with

$$
V^{*}(x, s, \lambda)<s \leq V_{\infty}(x, s) .
$$

For $\lambda>2 r(r-\mu) / \sigma^{2}$, it is clear that $V^{*}(x, s, \lambda) \leq s \leq V_{\infty}(x, s)$ for $x \leq a^{*} s$. To prove the statement for $x \geq a^{*} s>a s$, it suffices to show that $\left(a / a^{*}\right)^{\beta}(\alpha-r \bar{\alpha} /(r+\lambda)) \leq \alpha$ and $\left(a / a^{*}\right)^{\alpha}(r \bar{\alpha} /(r+\lambda)-\beta) \leq-\beta$. If we replace $a$ and $a^{*}$ by the expressions in (3) and (17), respectively, the first inequality becomes

$$
\left(\frac{\beta(r \bar{\alpha} /(r+\lambda)-\alpha)}{\alpha(r \bar{\alpha} /(r+\lambda)-\beta)}\right)^{\beta /(\alpha-\beta)}\left(\alpha-\frac{r \bar{\alpha}}{r+\lambda}\right) \leq \alpha,
$$

which is equivalent to

$$
\left(\alpha-\frac{r \bar{\alpha}}{r+\lambda}\right)^{\alpha /(\alpha-\beta)}\left(\frac{r \bar{\alpha}}{r+\lambda}-\beta\right)^{-\beta /(\alpha-\beta)} \leq \alpha^{\alpha /(\alpha-\beta)}(-\beta)^{-\beta /(\alpha-\beta)} .
$$

Define $f(x)=(\alpha-x)^{\alpha /(\alpha-\beta)}(x-\beta)^{-\beta /(\alpha-\beta)}$. It is sufficient to show that when $0 \leq x \leq \alpha$, we have $f(x) \leq f(0)$. This is obvious since

$$
f^{\prime}(x)=(-x)(\alpha-x)^{\alpha /(\alpha-\beta)-1}(x-\beta)^{-\beta /(\alpha-\beta)-1} \leq 0 .
$$

The second inequality can be verified in the same way.

Proposition 5. We have $V^{*}(x, s, \lambda) \rightarrow V_{\infty}(x, s)$ as $\lambda \rightarrow \infty$.

Proof. When $(x, s) \in\{(x, s), x \leq a s\}$, we have $V_{\infty}(x, s)=s$ and

$$
V^{*}(x, s, \lambda)=\frac{\lambda}{\lambda+r} s+\frac{r}{\lambda+r} s\left(\frac{x}{a^{*} s}\right)^{\bar{\alpha}} ;
$$

hence, as $\lambda \rightarrow \infty$, we have $V^{*}(x, s, \lambda) \rightarrow s=V_{\infty}(x, s)$.

When $(x, s) \in\{(x, s), x>a s\}$, since $a^{*} \rightarrow a$ there exists a $\lambda_{1} \geq 2 r(r-\mu) / \sigma^{2}$ such that $(x, s) \in\left\{(x, s), x \geq a^{*} s\right\}$ when $\lambda \geq \lambda_{1}$. Therefore,

$$
V^{*}(x, s, \lambda)=\frac{s}{\alpha-\beta}\left(\left(\frac{r \bar{\alpha}}{\lambda+r}-\beta\right)\left(\frac{x}{a^{*} s}\right)^{\alpha}-\left(\frac{r \bar{\alpha}}{\lambda+r}-\alpha\right)\left(\frac{x}{a^{*} s}\right)^{\beta}\right)
$$

and, hence, as $\lambda \rightarrow \infty$, we have

$$
V^{*}(x, s, \lambda) \rightarrow \frac{s}{\alpha-\beta}\left(-\beta\left(\frac{x}{a s}\right)^{\alpha}+\alpha\left(\frac{x}{a s}\right)^{\beta}\right)=V_{\infty}(x, s) .
$$




\section{Conclusion}

In this paper, we have solved an optimal stopping problem for a geometric Brownian motion in which the payoff function involves the running maximum and there is a constraint of random stopping. We could consider variants of the problem of determining (5), for instance the case in which $X_{t}$ is taken to be a Brownian motion instead of a geometric Brownian motion and the corresponding payoff function is taken to be $S_{\tau}-r \tau$ instead of $\mathrm{e}^{-r \tau} S_{\tau}$. It follows almost verbatim from the analysis in this paper that the solution structure remains the same.

\section{Appendix A. Proof of Theorem 1}

Our first claim is that $\mathrm{e}^{-r \tau_{n}} V_{0}\left(X_{\tau_{n}}, S_{\tau_{n}}\right)$ is a nonnegative, uniformly integrable supermartingale. This can be proved in three steps.

Step 1. From (9), we see that

$$
-(\lambda+r) V(x, s)+\mu x \frac{\partial V(x, s)}{\partial x}+\frac{1}{2} \sigma^{2} x^{2} \frac{\partial^{2} V(x, s)}{\partial x^{2}}+\lambda V_{0}(x, s)=0 .
$$

Now define

$$
Y_{T}=\mathrm{e}^{-(r+\lambda) T} V\left(X_{T}, S_{T}\right)+\int_{0}^{T} \lambda \mathrm{e}^{-(r+\lambda) t} V_{0}\left(X_{t}, S_{t}\right) \mathrm{d} t .
$$

Applying Itô's rule to $Y_{t}$, as in [16], it is easy to see that $Y_{T}$ is a martingale by recalling that $\mathrm{d} S_{t}=0$ (unless $X_{t}=S_{t}$ ) and $V_{s}(s, s)=0$ and by using Lemma 2 . Thus,

$$
\begin{aligned}
V(x, s) & =\mathrm{E}_{x, s}\left[Y_{0}\right] \\
& =\mathrm{E}_{x, s}\left[Y_{T}\right] \\
& =\mathrm{E}_{x, s}\left[\mathrm{e}^{-(r+\lambda) T} V\left(X_{T}, S_{T}\right)\right]+\mathrm{E}_{x, s}\left[\int_{0}^{T} \lambda \mathrm{e}^{-(r+\lambda) t} V_{0}\left(X_{t}, S_{t}\right) \mathrm{d} t\right] .
\end{aligned}
$$

Step 2. Note that $V(x, s) \leq K s$ uniformly for a constant $K$ when $r>\mu$. Therefore,

$$
\limsup _{T \rightarrow \infty} \mathrm{E}_{x, s}\left[\mathrm{e}^{-(r+\lambda) T} V\left(X_{T}, S_{T}\right)\right] \leq K \mathrm{e}^{-(r+\lambda) T} \mathrm{E}\left[S_{T}\right] \leq K \mathrm{e}^{-(r+\lambda) T}\left(s+x \mathrm{e}^{\mu T}\right) \rightarrow 0 .
$$

By taking $T \rightarrow \infty$ and using the monotone convergence theorem, we find that

$$
V(x, s)=\mathrm{E}_{x, s}\left[\int_{0}^{\infty} \lambda \mathrm{e}^{-(r+\lambda) t} V_{0}\left(X_{t}, S_{t}\right) \mathrm{d} t\right]
$$

Thus,

$$
V_{0}(x, s) \geq V(x, s)=\mathrm{E}\left[\int_{0}^{\infty} \lambda \mathrm{e}^{-(r+\lambda) t} V_{0}\left(X_{t}, S_{t}\right) \mathrm{d} t\right]=\mathrm{E}_{x}\left[\mathrm{e}^{-r G} V_{0}\left(X_{G}, S_{G}\right)\right],
$$

where $G$ is an exponential random variable with parameter $\lambda$.

Step 3. We claim that $\mathrm{e}^{-r \tau_{n}} V_{0}\left(X_{\tau_{n}}, S_{\tau_{n}}\right)$ is uniformly integrable. This is because $V_{0}\left(X_{t}, S_{t}\right)$ is bounded by $K S_{t}$ and there exists a $p>1$ for which $\sup _{0 \leq t<\infty} \mathrm{E}\left[\left|\mathrm{e}^{-r t} S_{t}\right|^{p}\right]<\infty$, following a more general result of [6]. 
We are now ready to prove the optimality of the value function, i.e. $V^{*}(x, s)=V(x, s)$. First, the optional sampling theorem [13] implies that

$$
V_{0}(x, s) \geq \mathrm{E}_{x, s}\left[\mathrm{e}^{-r \tau_{1}} V_{0}\left(X_{\tau_{1}}, S_{\tau_{1}}\right)\right] \geq \mathrm{E}_{x, s}\left[\mathrm{e}^{-r \tau_{N}} V_{0}\left(X_{\tau_{N}}, S_{\tau_{N}}\right)\right] \geq \mathrm{E}_{x, s}\left[\mathrm{e}^{-r \tau_{N}} S_{\tau_{N}}\right] .
$$

This, combined with (21), leads to

$$
\begin{aligned}
\mathrm{E}_{x, s}\left[\mathrm{e}^{-r \tau_{N}} S_{\tau_{N}}\right] & \leq \mathrm{E}_{x, s}\left[\mathrm{e}^{-r \tau_{N}} V_{0}\left(X_{\tau_{N}}, S_{\tau_{N}}\right)\right] \\
& \left.\leq V_{0}\left(X_{\tau_{1}}, S_{\tau_{1}}\right)\right] \\
& =\mathrm{E}\left[\int_{0}^{\infty} \lambda \mathrm{e}^{-(r+\lambda) t} V_{0}\left(X_{t}, S_{t}\right) \mathrm{d} t\right] \\
& =V(x, s) .
\end{aligned}
$$

By taking the supremum of all stopping times $\tau_{n}$, we find, for all $x \leq s$, that

$$
V(x, s) \geq V^{*}(x, s, \lambda) .
$$

The equality $V(x, s)=V^{*}(x, s, \lambda)$ now follows naturally from the strong Markov property of $\left(X_{t}, S_{t}\right)$, such that

$$
\begin{aligned}
\mathrm{E}_{x, s}\left[\mathrm{e}^{-r \tau^{*}} S_{\tau^{*}}\right] & =\mathrm{E}_{x, s}\left[\int_{0}^{\infty} \lambda \mathrm{e}^{-r \tau^{*}} \mathrm{E}_{x, s}\left[\mathrm{e}^{-r \tau^{*}} S_{\tau_{*}} \mid T_{1}=t\right] \mathrm{d} t\right] \\
& =\mathrm{E}_{x, s}\left[\int_{0}^{\infty} \lambda \mathrm{e}^{-(r+\lambda) t} \mathrm{E}_{X_{t}, S_{t}}\left[\mathrm{e}^{-r \tau_{0}^{*}} S_{\tau_{0}^{*}}\right] \mathrm{d} t\right] \\
& =\mathrm{E}\left[\int_{0}^{\infty} \lambda \mathrm{e}^{-(r+\lambda) t} V_{0}\left(X_{t}, S_{t}\right) \mathrm{d} t\right] \\
& =V(x, s),
\end{aligned}
$$

where $\tau_{0}^{*} \in B \cup\{0\}$ in augmenting the choice of stopping time at $t=0$.

\section{Acknowledgements}

We thank the anonymous referees for their very careful evaluation of our manuscript and for their insightful and constructive comments and suggestions.

\section{References}

[1] Benes, V. E., Shepp, L. and Witsenhauser, H. S. (1980). Some solvable stochastic control problems. Stochastics 4, 39-83.

[2] Chernoff, H. (1961). Sequential tests for the mean of a normal distribution. In Proc. 4th Berkeley Symp. Statist. Prob. Vol. 1, University of California Press, Berkeley, CA, pp. 79-91.

[3] Dubins, L. E., Shepp, L. And Shiryaev, A. (1993). Optimal stopping rules and maximal inequalities for Bessel processes. Theory Prob. Appl. 38, 226-261.

[4] Duffie, D. and Harrison, M. (1993). Arbitrage pricing of perpetual lookback options. Ann. Appl. Prob. 3, 641-651.

[5] Dupuis, P. And Wang, H. (2002). Optimal stopping with random intervention time. Adv. Appl. Prob. 34, 141157.

[6] Guo, X. (2001). An explicit solution to an optimal stopping time with regime switching. J. Appl. Prob. 38, 454-481.

[7] Guo, X. ANd Shepp, L. (2001). Some optimal stopping problems with non-trivial boundaries in pricing exotic options. J. Appl. Prob. 39, 1-12. 
[8] JACKA, S. D. (1991). Optimal stopping and best constants for Doob-like inequalities: the case $p=1$. Ann. Prob. 19, 1798-1821.

[9] JACKA, S. D. (1991). Optimal stopping and the American put. Math. Finance 1, 1-14.

[10] Karatzas, I. And Shreve, S. E. (1998). Brownian Motion and Stochastic Calculus (Graduate Texts Math. 113). Springer, New York.

[11] Krylov, N. V. (1980). Controlled Diffusion Process. Springer, New York.

[12] McKean, H. P. (1965). Appendix: A free boundary problem for the heat equation arising from a problem in mathematical economics. Industrial Manag. Rev. 6, 32-39.

[13] Revuz, D. ANd Yor, M. (1999). Continuous Martingales and Brownian Motion, 3rd edn. Springer, Berlin.

[14] Rogers, L. C. G. And Zane, O. (2000). A simple model of liquidity effects. In Advances in Finance and Stochastics, eds. K. Sandmann and P. Schoenbucher, Springer, Berlin, pp. 161-176.

[15] Shepp, L. and Shiryayev, A. N. (1993). The Russian option: reduced regret. Ann. Appl. Prob. 3, 631-640.

[16] Shepp, L. A. And Shiryaev, A. N. (1994). A new look at the "Russian Option". Theory Prob. Appl. 39, $103-119$.

[17] Van MoerbeKe, P. L. J. (1976). On optimal stopping and free boundary problems. Archive Rational Mech. Anal. 60, 101-148. 